\title{
COVID-19 vaccine (mRNA BNT162b2) and COVID-19 Infection-Induced Thrombotic Thrombocytopenic Purpura in Adolescents
}

\author{
Luna Vorster $^{1}$, Susan Kirk ${ }^{2}$, Eyal Muscal ${ }^{3}$, Jenny Despotovic ${ }^{4}$, Clay Cohen ${ }^{1}$, and Sarah \\ Sartain ${ }^{1}$ \\ ${ }^{1}$ Baylor College of Medicine \\ ${ }^{2}$ Baylor College of Medicine Department of Pediatrics \\ ${ }^{3}$ Baylor College of Medicine/Texas Children's Hospital \\ ${ }^{4}$ Texas Children's Hospital
}

November 24, 2021

\begin{abstract}
The mRNA COVID-19 vaccine and COVID-19 infection caused by the SARS-CoV-2 virus may be immunologic triggers for the development of thrombotic thrombocytopenic purpura (TTP). There is not yet literature that discusses TTP induced by COVID-19 vaccination or infection in pediatric or adolescent patients. We describe 4 adolescents presenting with TTP (both de novo and relapsed disease) following administration of the Pfizer COVID-19 vaccine or after COVID-19 infection. Our observations demonstrate that the Pfizer-BioNTech mRNA vaccine and COVID-19 infection can act as triggers for the development/relapse of both congenital and acquired TTP.
\end{abstract}

\section{Hosted file}

TTP Covid PBC_SES.pdf available at https://authorea.com/users/447851/articles/546773-covid19-vaccine-mrna-bnt162b2-and-covid-19-infection-induced-thrombotic-thrombocytopenicpurpura-in-adolescents 


\begin{tabular}{|c|c|c|c|c|c|c|c|c|c|}
\hline Patient & $\begin{array}{c}\text { Age } \\
\text { (years) }\end{array}$ & Sex & $\begin{array}{l}\text { Medical } \\
\text { history }\end{array}$ & Vaccine status & $\begin{array}{c}\text { Prior COVID } \\
\text { infection }\end{array}$ & TTP symptoms & Treatment & $\begin{array}{l}\text { Treatment Side } \\
\text { Effects }\end{array}$ & $\begin{array}{l}\text { Current clinical } \\
\text { status }\end{array}$ \\
\hline 1 & 19 & $\mathrm{~F}$ & TTP & $\begin{array}{l}\text { Pfizer- } \\
\text { BioNTech } \\
\text { mRNA vaccine } \\
\text { dose } 1\end{array}$ & $\begin{array}{l}\text { Not reported; SARS- } \\
\text { CoV-2 PCR not } \\
\text { detected at time of } \\
\text { relapse }\end{array}$ & $\begin{array}{l}\text { Bruising, } \\
\text { hematuria }\end{array}$ & $\begin{array}{l}\text { TPE } \times 4 \text { days } \\
\text { Methylprednisolone, } \\
\text { rituximab, } \\
\text { caplacizumab }\end{array}$ & None to-date & In remission \\
\hline 2 & 15 & $\mathrm{~F}$ & $\begin{array}{l}\text { Arrhythmia of } \\
\text { unknown } \\
\text { etiology, } \\
\text { previously on } \\
\text { metoprolol }\end{array}$ & $\begin{array}{c}\text { Pfizer- } \\
\text { BioNTech } \\
\text { mRNA vaccine } \\
\text { dose } 1\end{array}$ & $\begin{array}{l}\text { Not reported; SARS- } \\
\text { Cov-2 Anti-Spike } \\
\text { IgM positive after } \\
\text { vaccination }\end{array}$ & Fatigue, bruising & $\begin{array}{c}\text { TPE } \times 4 \text { days, } \\
\text { Methylprednisolone, } \\
\text { rituximab, FFP infusion }\end{array}$ & $\begin{array}{l}\text { Herpes zoster } \\
\text { infection, weight } \\
\text { gain }\end{array}$ & In remission \\
\hline 3 & 17 & $\mathrm{~F}$ & $\begin{array}{l}\text { Asthma, } \\
\text { NAFLD }\end{array}$ & Unvaccinated & $\begin{array}{l}\text { No clinical history; } \\
\text { SARS-CoV-2 IG } \\
\text { antibodies positive } \\
\text { on presentation }\end{array}$ & $\begin{array}{l}\text { Hemiparesis, } \\
\text { headache, fatigue, } \\
\text { juandice, } \\
\text { hematuria }\end{array}$ & $\begin{array}{l}\text { TPE } \times 5 \text { days, } \\
\text { rituximab, } \\
\text { caplacizumab }\end{array}$ & $\begin{array}{l}\text { Steroid induced } \\
\text { hyperglycemia } \\
\text { requiring insulin, } \\
\text { extremity tremors }\end{array}$ & In remission \\
\hline 4 & 17 & M & $\begin{array}{l}\text { ASD/VSD } \\
\text { repaired; } \\
\text { precocious } \\
\text { puberty } \\
\text { treated with } \\
\text { hormone } \\
\text { suppression }\end{array}$ & Unvaccinated & $\begin{array}{l}3 \text { weeks prior to } \\
\text { presentation with } \\
\text { symptomatic } \\
\text { infection; COVID } \\
\text { antibodies negative } \\
-3 \text { months after } \\
\text { initial presentation }\end{array}$ & $\begin{array}{l}\text { Initial hematuria; } \\
\text { representation } \\
\text { with jaundice, } \\
\text { pallor, nueurologic } \\
\text { abnormalities; } \\
\text { refractory }\end{array}$ & $\begin{array}{l}\text { TPE } \times 5 \text { days, } \\
\text { rituximab, prednisone, } \\
\text { cyclosporine, } \\
\text { caplacizumab, FFP } \\
\text { infusion }\end{array}$ & $\begin{array}{l}\text { Hypertension } \\
\text { during steroid } \\
\text { course }\end{array}$ & $\begin{array}{l}\text { Receiving } \\
\text { Koate-DVI } \\
\text { infusions } \\
\text { biweekly }\end{array}$ \\
\hline $\begin{array}{l}\text { FFP }=\text { fre } \\
\text { NAFLD } \\
\text { ASD }=a \\
\text { VSD }=\text { v }\end{array}$ & $\begin{array}{l}\text { zen } \\
\text { icot } \\
\text { ptal }\end{array}$ & boc & $\begin{array}{l}\text { topenic purpura } \\
\text { xchange } \\
\text { liver disease } \\
\text { ect }\end{array}$ & & & & & & \\
\hline
\end{tabular}


TABLE 2: Laboratory Results on Presentation of COVID-19 Vaccination or Infection-induced TTP

\begin{tabular}{|c|c|c|c|}
\hline Patient & Labs on presentation ${ }^{*}$ & ADAMTS13 & Comments \\
\hline 1 & $\begin{array}{c}\text { Hgb: } 12.4 \mathrm{gm} / \mathrm{dL} \\
\text { Plt: } 7 \times 10^{3} / \mathrm{uL} \\
\text { Abs retic: } 0.103 \times 10^{6} / \mathrm{uL} \\
\text { BiliU: } 5.3 \mathrm{mg} / \mathrm{dL} \\
\text { LDH: } 836 \mathrm{U} / \mathrm{L} \\
\text { C3: } 129 \mathrm{mg} / \mathrm{dL} \\
\text { C4: } 30 \mathrm{mg} / \mathrm{dL}\end{array}$ & $\begin{array}{c}\text { Activity }<5 \% \text {, inhibitor } 55 \% \text { [ref } \\
\text { range }<30 \%]\end{array}$ & $\begin{array}{l}\text { Schistocytes on } \\
\text { peripheral smear }\end{array}$ \\
\hline 2 & $\begin{array}{c}\text { Hgb: } 6.5 \mathrm{gm} / \mathrm{dL} \\
\text { Plt: } 33 \times 10^{3} / \mathrm{uL} \\
\text { Abs retic: } 0.286 \times 10^{6} / \mathrm{uL} \\
\text { BiliU: } 1.4 \mathrm{mg} / \mathrm{dL} \\
\text { LDH: } 354 \mathrm{U} / \mathrm{L} \\
\text { C3: } 130 \mathrm{mg} / \mathrm{dL} \\
\text { C4: not obtained }\end{array}$ & $\begin{array}{c}\text { Activity }<5 \% \text {, inhibitor } 25 \% \text { [ref } \\
\text { range }<30 \% \text { ] }\end{array}$ & $\begin{array}{l}\text { Schistocytes on } \\
\text { peripheral smear }\end{array}$ \\
\hline 3 & $\begin{array}{l}\text { Hgb: } 8.1 \mathrm{gm} / \mathrm{dL} \\
\text { PIt: } 5 \times 10^{3} / \mathrm{uL} \\
\text { Abs retic: } 0.522 \times 10^{6} / \mathrm{uL} \\
\text { BiliU: } 4.9 \mathrm{mg} / \mathrm{dL} \\
\text { LDH: } 2824 \mathrm{U} / \mathrm{L} \\
\text { C3: } 106 \mathrm{mg} / \mathrm{dL} \\
\text { C4: } 15 \mathrm{mg} / \mathrm{dL}\end{array}$ & $\begin{array}{l}\text { Activity }<5 \% \text {, inhibitor } 54 \\
\% \text { [ref range }<30 \%]\end{array}$ & $\begin{array}{l}\text { Schistocytes on } \\
\text { peripheral smear }\end{array}$ \\
\hline 4 & $\begin{array}{c}\text { Hgb: } 11.9 \mathrm{gm} / \mathrm{dL} \\
\text { Plt: } 352 \times 10^{3} / \mathrm{uL} \\
\text { Abs retic: } 0.082 \times 10^{6} / \mathrm{uL} \\
\text { BiliU: } 0.3 \mathrm{mg} / \mathrm{dL} \\
\text { LDH: } 465 \mathrm{UL} / \mathrm{L} \\
\text { C3: } 147 \mathrm{mg} / \mathrm{dL} \\
\text { C4: } 21 \mathrm{mg} / \mathrm{dL}\end{array}$ & $\begin{array}{c}\text { Activity }<5 \% \text {, inhibitor } 0.4 \text { [ref } \\
\text { range }<0.4 \text { ]; novel homozygous } \\
\text { variant in the ADAMTS13 gene, } \\
\text { NM_139025.4:c.1584+5G }>\text { A }\end{array}$ & $\begin{array}{c}\text { Note: labs from initial } \\
\text { encounter with TCH } \\
\text { Hematology; had } \\
\text { already received } \\
\text { treatment at prior } \\
\text { hospital }\end{array}$ \\
\hline \multicolumn{4}{|c|}{$\begin{array}{l}\text { Hgb=hemoglobin } \\
\text { Plt=platelets } \\
\text { Abs retic=absolute reticulocyte } \\
\text { BiliU=unconjugated bilirubin } \\
\text { LDH=lactate dehydrogenase } \\
\text { TCH=Texas Children's Hospital } \\
\text { TCH institutional reference ranges: Hgb } 10.6-13.5 \mathrm{gm} / \mathrm{dL}, \text { PIt } 186-353 \times 10^{3} / \mathrm{uL} \text {; Abs retic } 0.039-0.057 \times 10^{6} / \mathrm{uL} \text {; } \\
\text { BiliU < } 1.0 \mathrm{mg} / \mathrm{dL} \text {, LDH } 151-298 \mathrm{U} / \mathrm{L} \text {, C3 } 86-182 \mathrm{mg} / \mathrm{dL}, \mathrm{C} 417-51 \mathrm{mg} / \mathrm{dL} \text {. }\end{array}$} \\
\hline
\end{tabular}

